$\xi=-$ 圆

\title{
Design of Power System Stabilizer using Flower Pollination Algorithm
}

\author{
S. Venkateswarlu ${ }^{1}$, Janaki $\mathbf{M}^{1 *}$ and Thirumalaivasan $\mathbf{R}^{1}$ \\ ${ }^{1}$ School of Electrical Engineering, Vellore Institute of Technology, Vellore, Tamilnadu, India. \\ *Corresponding author E-mail: janaki.m@vit.ac.in
}

\begin{abstract}
The Power System Stabilizer (PSS) is a controller which is used to mitigate the instability of Low Frequency Oscillations (LFOs) in power systems. The condition of oscillatory instability can also cause the loss of generator synchronism. It is observed that the damping provided by PSS depends on the proper selection of its parameters. This paper presents the systematic method for the selection of PSS parameters using evolutionary nature inspired optimization technique called Flower Pollination Algorithm (FPA). FPA is employed for selecting the optimal parameters of PSS so as to mitigate the low frequency oscillations of generator rotor and thereby oscillatory instability. The system consists of Single Machine with PSS which is connected to Infinite Bus (SMIB) through a transmission line. The transient simulation validates the performance of the system with optimized PSS. The results show that PSS with FPA optimized parameters provides fast and stable response.
\end{abstract}

Keywords: Power System Stabilizer (PSS), Low Frequency Oscillations (LFOs), Flower Pollination Algorithm (FPA).

\section{Introduction}

The modern power systems with a long transmission lines are critically affected by low frequency oscillations particularly under stressed condition with random high load fluctuations. The occurrence of load fluctuations can initiate the swinging of generator rotor and hence low frequency oscillations originate. The ever present random load fluctuations can cause an adverse harmful effect on the rotor oscillations. If the damping of the system is not adequate (even if negative) then the adverse effect is shown in the long transmission line which threatens the power system stability and also the longevity. The range of LFO lies between 0.2 to 2.0 $\mathrm{Hz}$. The problem of LFOs aggravates further by the fast acting excitation system and high gain Automatic Voltage Regulator (AVR). Hence, it is noteworthy that damping of the system has to be improved in the low frequency range. PSS is the supplementary controller in the excitation system which helps to mitigate the problems of LFOs. It is a cost effective and satisfactory solution [1], [2].

There exists a considerable literature on the design of PSS to mitigate the LFOs and to enhance the system stability. The previous research has proposed various Meta heuristic and evolutionary optimization techniques which are applied to optimize the gain and time constants of PSS. The design of PSS based on Neural Network (NN) tuned Fuzzy Logic Controller (FLC) is discussed in [3]-[5], which is to mitigate rotor oscillations and enhance the small signal stability in power systems. The advantages of NN tuned Fuzzy Logic (FL) based PSS are simplification in learning capability and high computational speed. In [6], Bat Algorithm (BA) based PID-PSS is presented. The PID-PSS parameters are optimized using BA. The advantages of BA based PSS are to decrease the settling time, reduce the error value and gives superior performance.
The tuning of PSS parameters by using a recently proposed algorithm called Oppositional Gravitational Search Algorithm (OGSA) is done in [7], which is used to mitigate LFOs and improve dynamic stability of the system. The design of PSS based on Genetic Algorithm (GA) is reported in [8], which is used to suppress the rotor oscillations, enhance the settling time and overshoot. The optimization of PSS parameters using Particle Swarm Optimization (PSO), Bacterial Foraging Algorithm (BFA) and Gravitational Search Algorithm (GSA) is reported in [9], [10] and [11] respectively.

The above stated optimization techniques have some drawbacks, such as consume more computational time, complexity, encoded parameters requires more capacity and results with less accuracy. These drawbacks can be eliminated with flower pollination algorithm [12], [13].

In the year 2012, Xin-she-yang has proposed an optimization algorithm based on natural pollination in flowers [14]. In this algorithm, the process of pollination takes place through biotic cross pollination and abiotic self pollination. Among the two pollination processes, the biotic cross pollination is considered as global pollination. The best features of FPA are the global survey and local manipulation in the same iteration and randomness in every iteration by way of abiotic pollination. Hence, the optimization using FPA can ensure the global minimum or maximum of the problem. In this paper, a systematic selection of PSS parameters using flower pollination algorithm is presented. The objective of this paper is to improve the system stability and optimize the PSS parameters using FPA. The performance of the system with optimized PSS parameters is validated through the transient simulation under large disturbance.

The sections of this paper are organized as follows. The modelling of the power system is presented in Section 2. The design of PSS is explained in Section 3. The results and discussion are described in Section 4. At last, the conclusion is presented in Section 5. 


\section{Modelling of the Power System}

The power system model schematic diagram is shown in Figure 1. It includes synchronous generator, PSS, automatic voltage regulator, transformer, transmission line and infinite bus. The terminal voltage of the synchronous generator is denoted by $\mathrm{V}_{\mathrm{t}}$ and the voltage of the infinite bus is denoted by $\mathrm{E}_{\mathrm{b}}$. The resistance and reactance of the transmission line are denoted by $R_{e}$ and $X_{e}$ respectively. The AVR and the excitation system are used for the controlling the terminal voltage of the synchronous generator which is indicated by $\mathrm{V}_{\mathrm{t}}$.

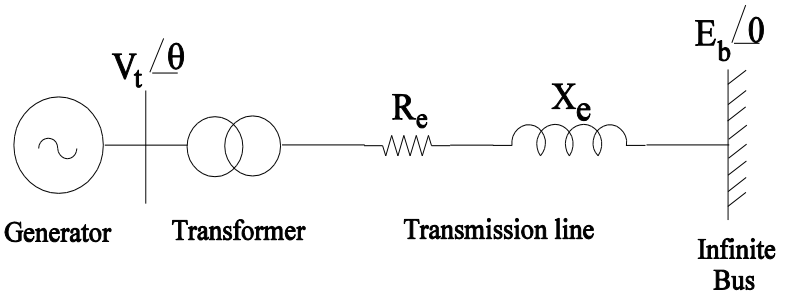

Fig. 1: Structure of Power System Model [1]

\subsection{Structure of Power System Stabilizer}

The purpose of PSS is to enhance the damping torque so has to overcome the negative damping provided by high gain, fast acting excitation system. The suitable input signal of PSS for this purpose is the generator speed deviation signal $(\Delta \omega)$ [1], [2]. The structure of PSS is shown in Figure 2.

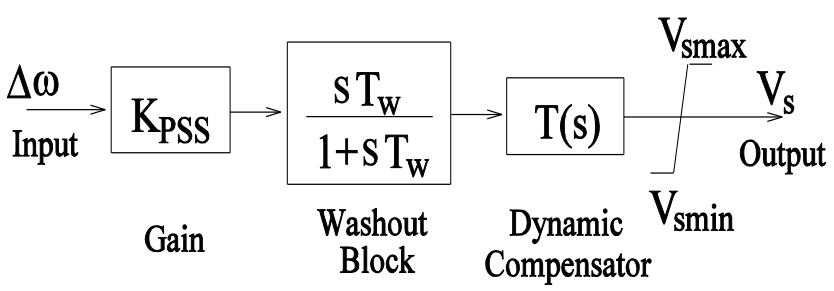

Fig. 2: Structure of Power System Stabilizer [1]

The PSS primarily consists of four blocks namely: gain, washout block, dynamic compensator and torsional filter. The torsional filter is not considered here. The output of PSS $\left(\mathrm{V}_{\mathrm{s}}\right)$ is given to the AVR as an input signal.

\subsubsection{Gain}

The damping of low frequency oscillations which corresponds to swing mode depends on the gain $\left(\mathrm{K}_{\mathrm{PSS}}\right)$ of PSS. Hence, the optimal value of gain must be chosen so has to obtain the maximum damping of swing mode.

\subsubsection{Washout Block}

The output signal of PSS may contain steady state bias. In order to remove the steady state bias, the high-pass filter is used in PSS which is called as washout block. The time constant $\left(\mathrm{T}_{\mathrm{W}}\right) \mathrm{im}$ proves the first swing stability of rotor and terminal voltage response.

\subsubsection{Dynamic Compensator}

In general, the constants of compensator block are chosen to provide suitable phase lead compensation in 0 to $3.5 \mathrm{~Hz}$. Also, $\mathrm{T}_{1}>$ $\mathrm{T}_{2}$ and $\mathrm{T}_{3}>\mathrm{T}_{4}$.

The transfer function of the dynamic compensator is given as follows
$\mathrm{T}(\mathrm{s})=\frac{\left(1+\mathrm{sT}_{1}\right)\left(1+\mathrm{sT}_{3}\right)}{\left(1+\mathrm{sT}_{2}\right)\left(1+\mathrm{sT}_{4}\right)}$

The complete transfer function of PSS is given as

$\operatorname{PSS}(\mathrm{s})=\mathrm{K}_{\mathrm{PSS}} \frac{\mathrm{sT}_{\mathrm{W}}\left(1+\mathrm{sT}_{1}\right)\left(1+\mathrm{sT}_{3}\right)}{\left(1+\mathrm{sT}_{\mathrm{W}}\right)\left(1+\mathrm{sT}_{2}\right)\left(1+\mathrm{sT}_{4}\right)}$

Where

$\mathrm{K}_{\mathrm{PSS}}, \mathrm{T}_{\mathrm{W}}, \mathrm{T}_{1}, \mathrm{~T}_{2}, \mathrm{~T}_{3}$ and $\mathrm{T}_{4}$ are gain, time constants of washout and compensator blocks respectively.

\section{Design of Power System Stabilizer}

The objective of PSS is to mitigate the LFOs and thereby enhance the stability of the power system. The improvement in the damping and stability can be achieved by properly selecting the parameters of PSS. Here, the gain and time constants of PSS are selected using flower pollination algorithm with the aim of improving the system damping. In the following subsections, the FPA optimization technique and the formulation of objective function are explained.

\subsection{Flower Pollination Algorithm based Optimization Technique}

FPA is an evolutionary optimization technique where the algorithm is based on nature inspired population. Surviving of best fittest plants is the main objective of the FPA to give the optimal reproduction of plants. Flower pollination is the process where pollens are transferred from one flower to the other giving rise to reproduction of the flowering plants. Flower pollination consists of two process namely: abiotic or self pollination and biotic or cross pollination. Self pollination occurs within same species of flowering plants and the pollinating medium is wind. In case of cross pollination, it occurs between the plants of different species at long distance and the pollinating mediums are bats, bees and birds. The pollinators of cross pollination perform Levy flight. The biotic cross pollination and abiotic self pollination are categorized as local pollination and global pollination respectively. Also, it is worthwhile to mention that $90 \%$ of pollination takes place through biotic cross pollination. Switching action between local and global pollination is done by probability switch $\mathrm{P}$. The range of probability switch $(\mathrm{P})$ is lies in between 0 to 1 [12]-[14].

The four rules in FPA are as follows:

Rule 1: Biotic or cross-pollination is categorized as global pollination. The pollinators carrying pollen follow Levy flights.

Rule 2: Abiotic form of self pollination is categorized as local pollination.

Rule 3: Insects act as pollinators introduce loyalty to flowers resulting into the growth of probability to generate new individuals, the probability is proportional to the similarity between two flowers taking part into the process of pollination.

Rule 4: Probability switch $p \in[0,1]$ is the controlling factor between global pollination and local pollination, somewhat biased towards local pollination.

The following steps are involved in FPA:

Step-1: Setting the FPA parameters such as the number of generation $(\mathrm{N})$, population size $(\mathrm{n})$ and switch probability $(\mathrm{p})$.

Step-2: Random initialization of the parameters $X_{i}$, under consideration takes place for the assumed population size $\mathrm{N}$. The fitness value is evaluated by calculating the objective function with every solution $\left(\mathrm{X}_{\mathrm{i}}\right)$.

Step-3: The process of global pollination starts with random number $r$ for each solution $\left(X_{i}\right)$, where $r$ in $[0,1]$.

Step-4: If $r<p$, the Le'vy distribution is adopted to generate the new solution as 


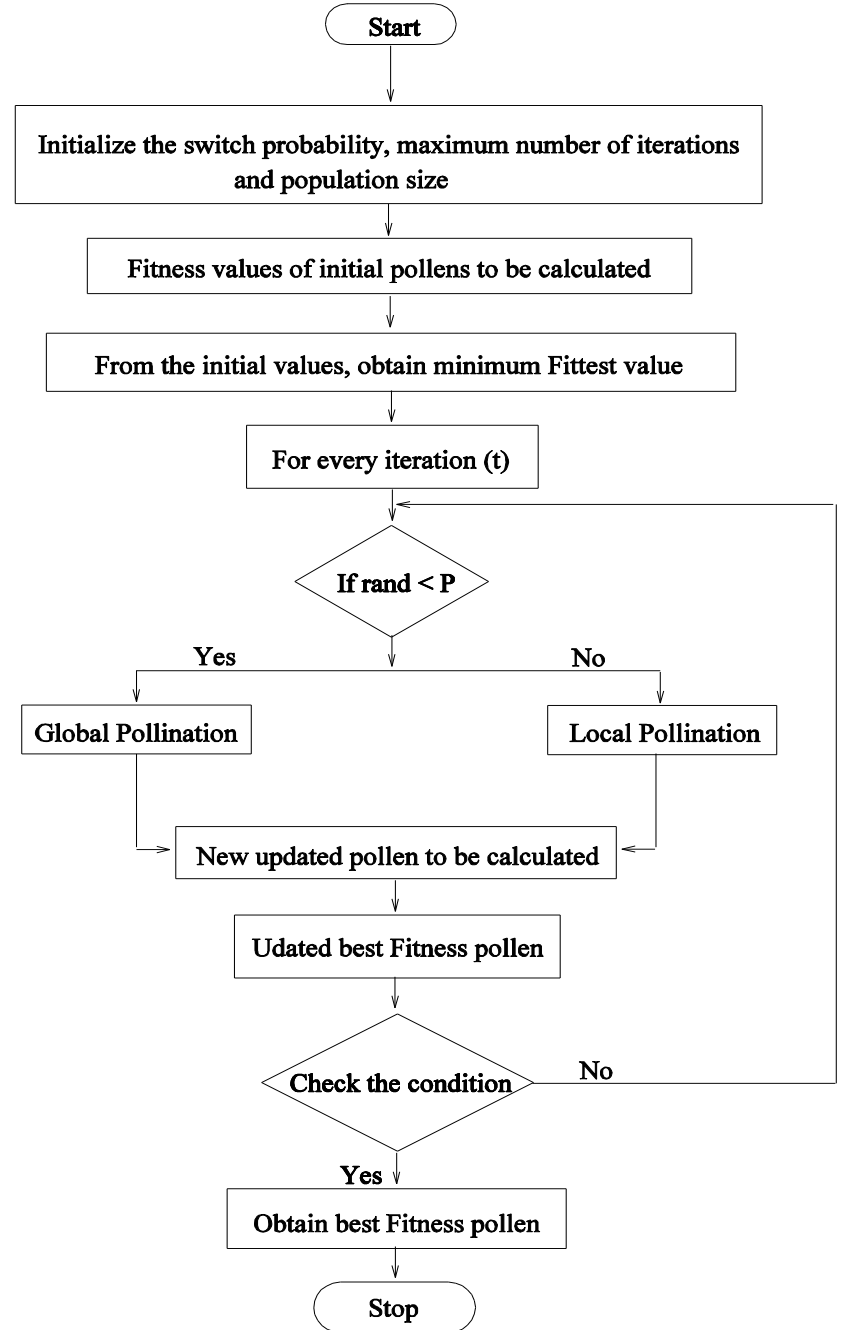

Fig. 3: FPA Flowchart

$\mathrm{X}_{\mathrm{i}}^{\mathrm{t}+1}=\mathrm{X}_{\mathrm{i}}^{\mathrm{t}+1}+\mathrm{L}\left(\mathrm{X}_{\mathrm{i}}^{\mathrm{t}}-\mathrm{g}^{*}\right)$

Where

$\mathrm{L}$ is a Le'vy flight and $\mathrm{L}>0$. The Le'vy flight is determined using

$\mathrm{L} \approx \frac{\lambda \Gamma(\lambda) \sin (\Pi \lambda / 2)}{\Pi} \frac{1}{\mathrm{~S}^{1+\lambda}}, \quad\left(\mathrm{S}>>\mathrm{S}_{0}>0\right)$

Step-5: Else, the local pollination takes place by producing random number $\varepsilon$, where $\varepsilon$ in $[0,1]$.

Step-6: Each solution $\left(\mathrm{X}^{\mathrm{t}}+1\right)$ is calculated and the population is updated according to fitness value.

Step-7: Sorting of solution and the newest best solution $\mathrm{g}^{*}$ is found.

Step-8: Step-3 to step-7 are repeated till the termination condition is satisfied. At the end, the best solution is presented.

The non-linear optimization problems can be solved by employing FPA. FPA yields better performance compared to other optimization techniques. FPA gives numerous following advantages over conventional optimization techniques; such as, quick convergence, minimum complexity, superior computational speed and better performance. The flowchart of FPA is considered for the controller parameter optimization is shown in Figure 3.

\subsection{Formulation of Objective Function}

The optimal set of PSS parameters is obtained using FPA. The primary objective in the design of PSS is to improve the system damping which can reduce the overshoot in rotor oscillations and suppress the oscillations in less settling time. Also, the stability of the system to be maintained.

Considering these facts, the objective function $\mathrm{E}$ is formulated as:

SumSquaredError $(E)=\sum_{n} \mathrm{e}^{2}$

Where

$\mathrm{e}=\mathrm{T}_{\mathrm{m}}-\mathrm{T}_{\mathrm{e}}$

$\mathrm{n}=1,2, \ldots \ldots \ldots \mathrm{m}$

$\mathrm{m}$ is size of $\mathrm{T}_{\mathrm{m}}$ array with transient simulation carried out for 2 sec.

$\mathrm{T}_{\mathrm{m}}$ is the mechanical torque and

$\mathrm{T}_{\mathrm{e}}$ is the electrical torque.

The objective function $\mathrm{E}$ is the sum of square of the difference between the mechanical and electrical torque, while maintaining the stability of the system. When the difference between the mechanical and electrical torque reduces, the transient performance of the generator rotor and thus the system can be improved. Hence, to achieve the desired performances, the optimization statement is structured as

Minimize E

Subjected to the condition that

Real part of all eigenvalues $<0$

The boundary limits of PSS parameters are given as

$$
\begin{array}{ll}
\mathrm{K}_{\mathrm{PSS}_{\text {min }}} \leq \mathrm{K}_{\mathrm{PSS}} \leq \mathrm{K}_{\mathrm{PSS}_{\text {max }}} & \mathrm{T}_{\mathrm{W}_{\text {min }}} \leq \mathrm{T}_{\mathrm{W}} \leq \mathrm{T}_{\mathrm{W}_{\text {max }}} \\
\mathrm{T}_{1_{\text {min }}} \leq \mathrm{T}_{1} \leq \mathrm{T}_{1_{\text {max }}} & \mathrm{T}_{2_{\text {min }}} \leq \mathrm{T}_{2} \leq \mathrm{T}_{2_{\text {max }}} \\
\mathrm{T}_{3_{\text {min }}} \leq \mathrm{T}_{3} \leq \mathrm{T}_{3_{\text {max }}} & \mathrm{T}_{4_{\text {min }}} \leq \mathrm{T}_{4} \leq \mathrm{T}_{4_{\max }}
\end{array}
$$

The range of PSS parameters is assessed from the locus of eigenvalues. The trajectories of eigenvalues for the variation in controller parameters are shown in Figure 4. We observe that for the large values of time constants $\left(\mathrm{T}_{1}\right.$ and $\left.\mathrm{T}_{3}\right)$, locus of eigenvalues are moving to right side of complex plane, hence the damping reduces and the eigenvalues are unstable. While the increase in other parameters shifts the locus of eigenvalues to left side of complex plane. We tune the PSS parameters to improve the transient performance of system while maintaining the stability. Hence, from the trajectories of eigenvalues and the realistic values of PSS parameters, we choose the upper and lower boundaries for controller parameters. Using FPA, the optimal parameters are obtained as:

$\mathrm{K}_{\mathrm{PSS}}=5.5, \mathrm{~T}_{\mathrm{W}}=11, \mathrm{~T}_{1}=0.15, \mathrm{~T}_{2}=0.04, \mathrm{~T}_{3}=0.15, \mathrm{~T}_{4}=0.04$

\section{Results and Discussion}

The performance of the system with FPA optimized parameters for PSS is determined by transient simulation and eigenvalue analysis. In transient simulation, the step change in the reference value of mechanical torque is applied at $0.5 \mathrm{sec}$ and removed at $1.0 \mathrm{sec}$.

In the FPA optimization process of PSS parameters, the best minimum value of objective function for different iteration and population sizes are plotted in Figure 5 and Figure 6.

From Figure 5 and Figure 6 we observe that the value of objective function converges to best minimum in less number of iterations with increase in population size. The optimal values of controller parameters are considered to be the values corresponding to the best minimum value of objective function.

The location of eigenvalues in complex plane is plotted with optimal PSS parameters in Figure 7. In Figure 7, all the eigenvalues are on left half of the complex plane with optimal PSS parameters indicating that the system is stable.

The response of rotor angle with optimal PSS parameters for the step change in mechanical torque is plotted Figure 8. 

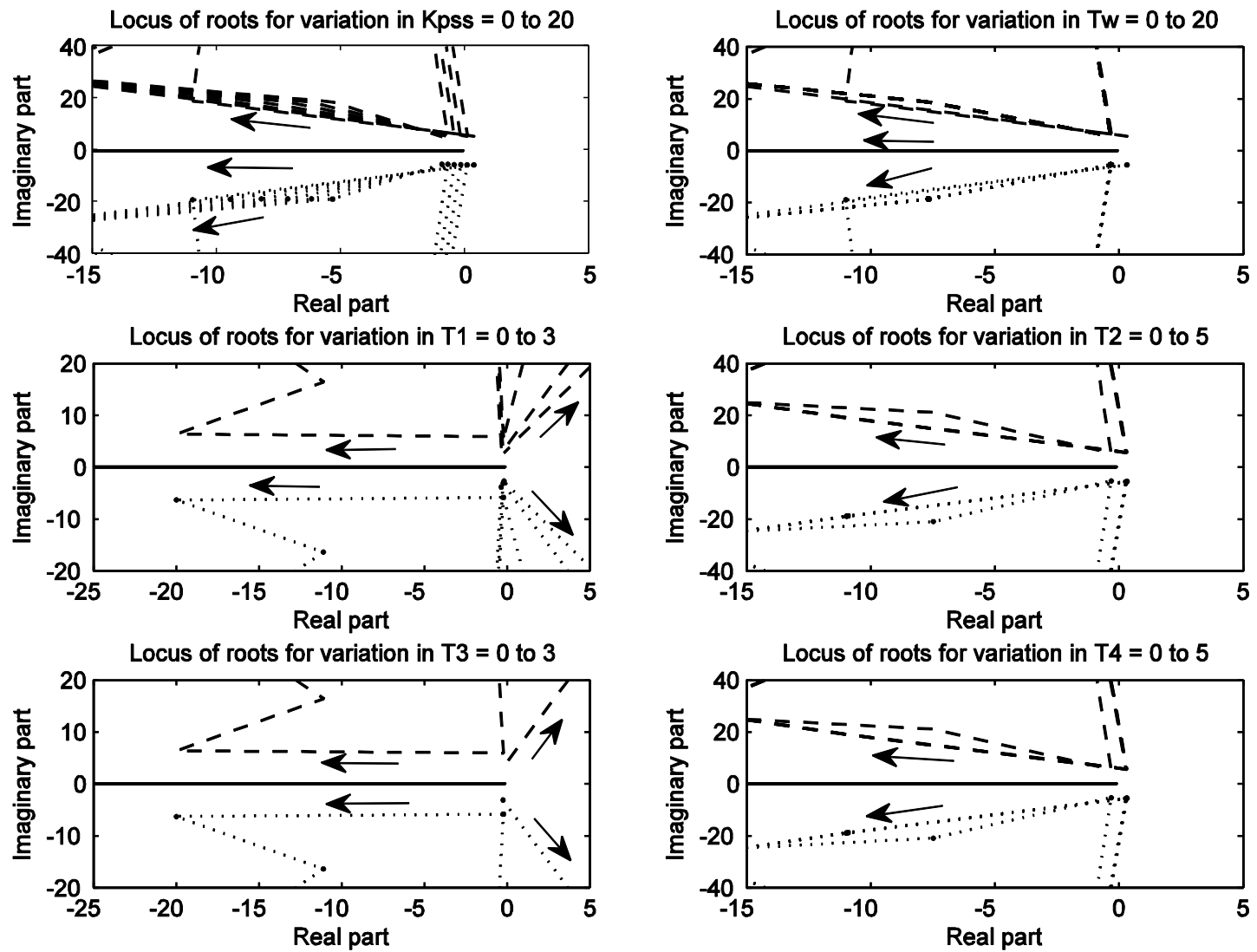

Fig. 4: Movement of the eigenvalues with the variation of PSS parameters

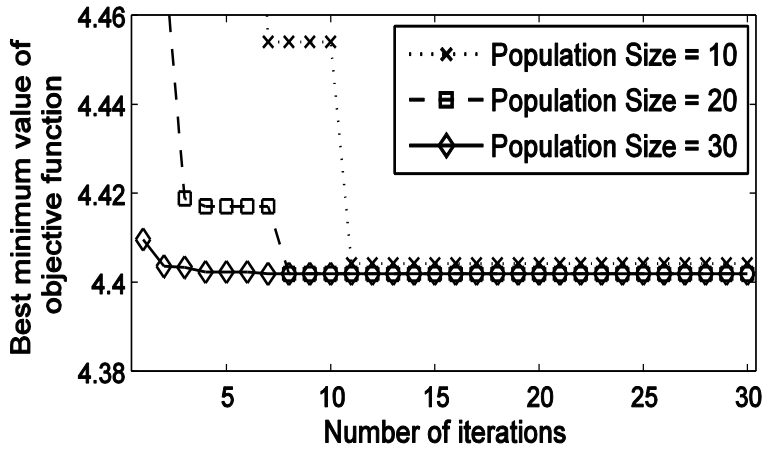

Fig. 5: Best minimum value of objective function in every iteration for different population size with 30 iterations

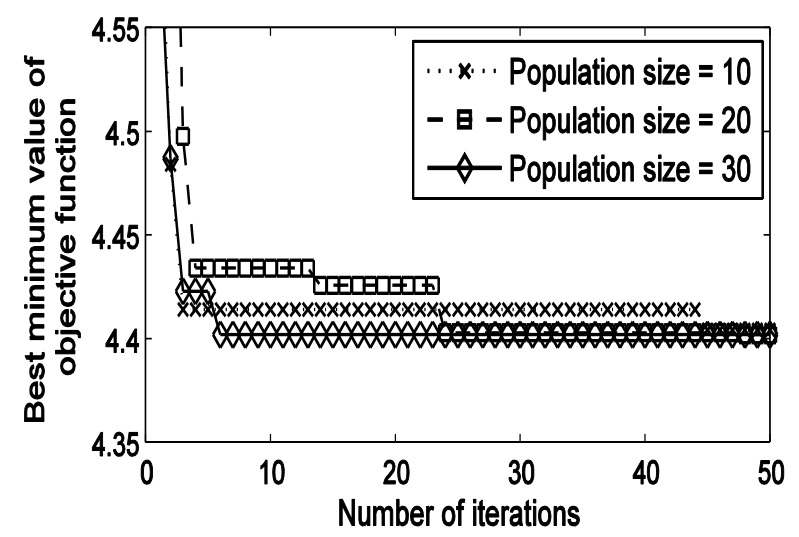

Fig. 6: Best minimum value of objective function in every iteration for different population size with 50 iterations

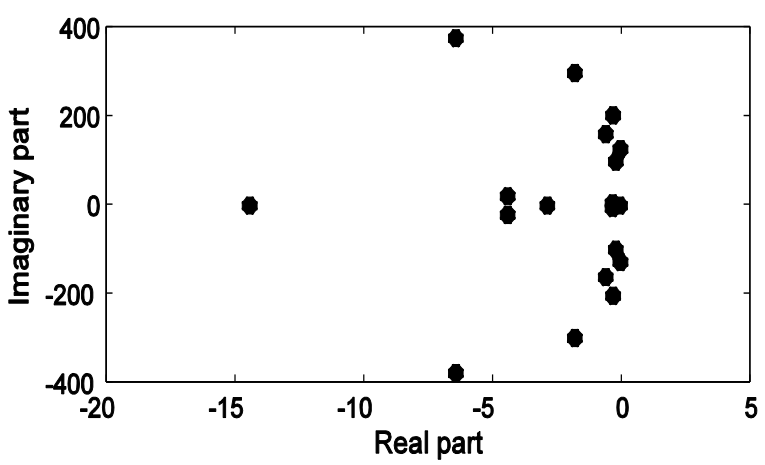

Fig. 7: Location of eigenvalues in complex plane with optimal PSS parameters

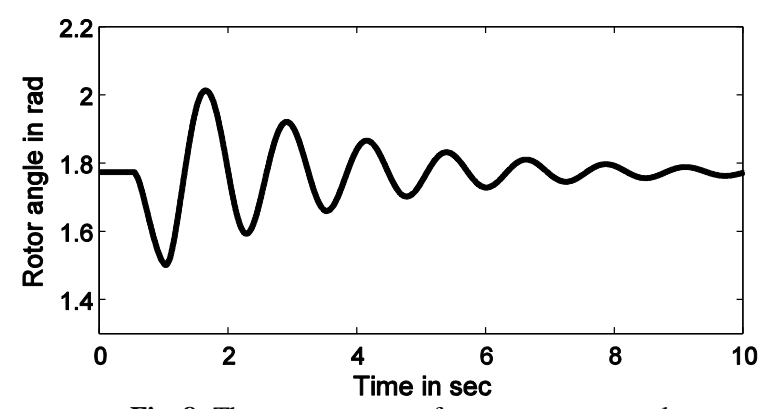

Fig. 8: The step response of generator rotor angle

In Figure 8, the oscillations in generator rotor reduce with time and reaches steady state value. Hence, it is evident that the application of FPA to optimize the PSS parameters ensures the stability of the system. 


\section{Conclusion}

In this paper, we have presented a systematic concept for the selection of PSS parameters based on FPA and to mitigate the LFOs of generator rotor. The FPA based optimization technique is used to optimize the controller parameters which ensures that the system is stable at the operating system conditions. The stability of the system is evident from the location of eigenvalues. The transient simulation results show that the rotor oscillations can be suppressed and reaches to steady state. The advantage of FPA is the fast convergence with less complexity.

\section{References}

[1] K. R. Padiyar, "Analysis of Subsynchronous Resonance in power systems", Boston: Kluwer Academic Publications, (1999).

[2] K. R. Padiyar, "Power System Dynamics-Stability and Control", Second Edition, Hyderabad, India: B.S.Publications, (2000).

[3] P. K. Arun Kumar, S. Vivekanandan, C. Krishna Kumar and V. Kumar Chinnaiyan, "Neural Network Tuned Fuzzy Logic Power System Stabilizer Design for SMIB", in Proc International Conference on Contemporary Computing and Informatics (IC3I), (2016), pp. 446-451.

[4] N. Hosseinzadeh and A. Kalam, "A Rule-Based Fuzzy Power System Stabilizer Tuned by a Neural Network", IEEE Trans. on Ener. Conv., vol. 14, no. 3, (1999), pp. 773-776.

[5] N. Hosseinzadeh and A. Kalam, "A Neuro-Fuzzy Power System Stabilizer", in Proc. International Conference on Industrial Electronics, Control, and Instrumentation (IECON), vol. 1, (1996), pp 608-613.

[6] K. Sambariya and R. Gupta, "Effective PID-PSS Design using Bat Algorithm for SMIB Power System", in Proc. International Conference on Power Systems (ICPS), (2016), pp. 1-6.

[7] S. Paul and P. K. Roy, "Optimal Design of Power System Stabilizer using Oppositional Gravitational Search Algorithm", in Proc. International Conference on Non Conventional Energy (ICONCE), (2014), pp. 282-287.

[8] P. R. Gandhi and S. K. Joshi, "Design of PID Power System Stabilizer using GA for SMIB System: Linear and Non-Linear Approach", in Proc. International Conference on Recent Advancements in Electrical, Electronics and Control Engineering (ICONRAEeCE), (2011), pp. 319-323.

[9] Pothula Jagadeesh and M Sai Veerraju, "Particle Swarm Optimization based Power System Stabilizer for SMIB System", in Proc. International Conference on Emerging Trends in Engineering, Technology and Science (ICETETS), (2016), pp. 1-6.

[10] K. Abdul hameed and S Palani, "Robust Design of Power System Stabilizer using Bacterial Foraging Algorithm", Archives of Electrical Engineering, vol. 62(1), (2013), pp. 141-152.

[11] Akash Saxena, Bhanu Pratap Soni and Vikas Gupta, "Minimax Approximation Synthesis in PSS Design by Embedding Gravitational Search Algorithm", in Proc. International Conference on Advanced Communication Control and Computing Technologies (ICACCCT), (2014), pp. 929-934

[12] J. Prasanth Ram, Babu T S and N. Rajasekar, "FPA based approach for solar maximum power point tracking", in Proc. International Conference in Power Systems, (2016), pp. 1-6.

[13] J. Prasanth Ram and N. Rajasekar, "A Novel Flower Pollination Based Global Maximum Power Point Method for Solar Maximum Power Point Tracking", IEEE Trans. on Power Electronics, vol. 32, No. 11, (2017), pp. 8486-8499.

[14] Yang, Xin-She, "Nature-Inspired Optimization Algorithms", Elsevier, (2014). 